\title{
VALIDAÇÃO DE METODOLOGIA PARA O TESTE DE GERMINAÇÃO EM SEMENTES DE NABO FORRAGEIRO (Raphanus sativus L. var. oleiferus) ${ }^{1}$
}

\author{
VERÔNICA YUMI KATAOKA², MARIA LAENE MOREIRA DE CARVALHO ${ }^{3}$, \\ MARCELO SILVA DE OLIVEIRA ${ }^{4}$, CARLA MASSIMO CALDEIRA ${ }^{5}$
}

\begin{abstract}
RESUMO - Para várias espécies vegetais, testes como o de germinação não tem seus procedimentos descritos nas Regras para Análise de Sementes (RAS) utilizadas no Brasil pela falta de padronização e validação metodológica. Dentre elas, o nabo forrageiro, que tem sido considerada uma cultura promissora para a produção de biodiesel, tem sua germinação avaliada por metodologia recomendado para a espécie Raphanus sativus, mas tem especificidade de variedade (var oleiferus) com características de plantas e sementes, que diferem das demais variedades da espécie. Para assegurar a padronização de metodologias para o comércio internacional de sementes é necessário estabelecer critérios para validação de metodologia, cuja descrição deve ser clara e completa com procedimentos que propiciem exatidão, robustez, precisão (reprodutibilidade e repetibilidade). O objetivo neste estudo foi avaliar o processo de validação de duas metodologias para o teste de germinação em sementes de nabo forrageiro, utilizando para as análises estatísticas o procedimento padrão da ISTA, bem como técnicas complementares. Os testes de germinação foram realizados em oito laboratórios com cinco lotes, utilizando substrato areia e papel em temperatura alternada $20-30{ }^{\circ} \mathrm{C}$. As técnicas estatísticas foram utilizadas para: verificar a homogeneidade dos lotes (teste $\mathrm{H}$ ), identificar a presença de valores discrepantes (método de Hampel) e outliers nas variâncias (teste de Levene para média); avaliar os efeitos de laboratórios e lotes (Análise de Variância); verificar a repetibilidade, reprodutibilidade, exatidão e robustez (limites críticos de repetibilidade, reprodutibilidade, estatísticas h e k de Mandel), comparar as diferentes metodologias de germinação (testes F) e reavaliar o nível de qualidade dos lotes (Análise Discriminante). Tanto no aspecto estatístico, como fitotécnico, as metodologias para o teste de germinação em sementes de nabo forrageiro com a utilização do substrato areia e papel, temperatura alternada $20-30{ }^{\circ} \mathrm{C}$ podem ser considerados validadas, pois apresentam exatidão, robustez e precisão adequadas.
\end{abstract}

Termos para indexação: validação de metodologias, testes estatísticos, nabo forrageiro.

\section{VALIDATION OF METHODOLOGIES FOR GERMINATION TEST IN OIL RADISH SEED (Raphanus sativus 1. var. oleiferus)}

\begin{abstract}
For several vegetable species, there is no procedure in the Rules for Seed Testing used in Brazil for tests such as germination, due to lack of standardization and methodological validation. Among these species is oil radish, a promising crop for bio-diesel production, whose germination is assessed by the methodology recommended for Raphanus sativus, although it
\end{abstract}

${ }^{1}$ Submetido em 13/04/2009. Aceito para publicação em 26/08/2010. Parte da Tese de Doutorado do primeiro autor apresentado a UFLA/MG.

${ }^{2}$ Eng. Agr., Dr, Professora Doutora do programa de Pós-graduação em Educação Matemática da Universidade Bandeirante de São Paulo. Av. Braz Leme, 3029, São Paulo, SP, Cep:02022-011. E-mail:veronicayumi@terra.com.br.

${ }^{3}$ Eng. Agr., Dr, Professora do Departamento de Agricultura da Universidade Federal de Lavras, Cx Postal 3037, Lavras, Minas Gerais,
Cep:37200-000. E-mail:mlaenemc@ufla.br.

${ }^{4}$ Eng. Agrícola, Dr., Professor Associado do Departamento de Ciências Exatas Agricultura da Universidade Federal de Lavras, Cx Postal 3037, Lavras, Minas Gerais, Cep:37200-000.E-mail:marcelo.oliveira@ufla.br

${ }^{5}$ Eng Agr., Doutoranda, Departamento de Agricultura da Universidade Federal de Lavras, Cx Postal 3037, Lavras, Minas Gerais, Cep:37200000.E-mail:carlaufla@com.br. 
has varietal specificity (var. oleiferus) with plant and seed characteristics that differ from other varieties of the species. To ensure standardization of methodologies for the international seed trade, The International Seed Testing Association (ISTA), establishes criteria for methodology validation, whose description should be clear and complete with procedures that should be accurate, robust and precise (reproducibility and repeatability). In this the study aims was to evaluate the validation process of two methodologies for the germination test in oil radish seeds, using the ISTA standard procedure, as well as complementary techniques, for statistical analyses. Germination tests were done in eight laboratories with five lots, using sand and paper substrates with alternating temperatures of 20-30 ${ }^{\circ} \mathrm{C}$. Statistical techniques were used to verify the homogeneity of lots ( $\mathrm{H}$ test), identify the presence of discrepant values (Hampel method) and outliers in variances (Levene's test for average); assess the effects of laboratories and lots (analysis of Variance); verify repeatability, reproducibility, accuracy and robustness (critical limits of repeatability, reproducibility, Mandel's h and k statistics); compare different germination methods (F tests); and reevaluate the quality level of lots (Discrimination Analysis). Both in statistical and phytotechnical aspects, the methodologies for the germination test in oil radish seeds with sand and paper substrates and alternating temperatures of $20-30{ }^{\circ} \mathrm{C}$ can be consider validated, as they demonstrated accuracy, robustness, repeatability and reproducibility.

Index Terms: validation of methodology, statistical tests, oil radish.

\section{INTRODUÇÃO}

Uma das condições para a produção de grãos de nabo forrageiro em larga escala é a produção de sementes em quantidade e qualidade suficientes para viabilizar o cultivo. Grãos de nabo forrageiro têm se revelado uma opção promissora como matéria-prima para a produção do biodiesel, por possuir alto rendimento em óleo de baixa viscosidade, com teor médio estimado de $35 \%$. O desafio atual é a produção de sementes de alta qualidade que possibilite aumentar a produtividade dessa cultura e, por conseguinte, viabilizar o seu uso como matériaprima competitiva e rentável no mercado de produção de biodiesel.

Para averiguar a qualidade das sementes são realizados testes, como o de germinação, realizado sob condições ideais e artificiais que permitem a manifestação do máximo potencial fisiológico do lote (AOSA, 1983). O teste de germinação tem seus procedimentos descritos nas Regras para Análise de Sementes -RAS (Brasil, 1992) utilizadas no Brasil para maioria das espécies comerciais, no entanto, apesar de haver recomendação para a espécie Raphanus sativus não existe um procedimento específico para variedade oleiferus, que apresenta características distintas em relação a outras variedades comerciais da mesma espécie.

Nery et al. (2007) e Nery (2008) realizaram testes de germinação para sementes de nabo forrageiro visando definir a temperatura ótima e o substrato ideal para avaliação da qualidade de sementes das cultivares CATI AL-1000 e IPR 116. Estes autores verificaram que a temperatura alternada de $20-30{ }^{\circ} \mathrm{C}$ e uso do substrato areia seriam as condições ideais para avaliar a qualidade das sementes de nabo forrageiro. Apesar destas pesquisas já apresentarem indicativos de uma metodologia adequada para o teste de germinação em sementes de nabo forrageiro, segundo a literatura internacional (Eurachem, 1998; Sheppard e Cockerell, 2000; ISTA, 2007), a designação de um teste ou metodologia deve basear-se em princípios de metrologia. Dessa forma, a indicação de um determinado teste deve ser precedida de procedimentos de validação metodológica.

A International Seed Testing Association (ISTA), para assegurar a padronização de metodologias para o comércio internacional de sementes, estabeleceu critérios de validação de metodologias em um protocolo elaborado em 2002. Este programa consiste em uma avaliação crítica para assegurar que a descrição da metodologia seja clara e completa, e que o procedimento forneça exatidão, robustez, precisão (reprodutibilidade e repetibilidade) dos resultados em concordância com as especificações estabelecidas pela própria metodologia.

Para a avaliação do processo de validação dessas metodologias, já existe uma recomendação da ISTA para 
o desenvolvimento de uma análise padrão, que considera a utilização de algumas técnicas estatísticas, mas que não restringe a proposição de novos procedimentos (ISTA, 2007).

O objetivo nesse trabalho foi validar duas metodologias para o teste de germinação em sementes de nabo forrageiro (Raphanus sativus var. oleiferus), utilizando como substrato a areia ou papel. Além disso, avaliar o uso de Técnicas estatísticas complementares ao procedimento padrão da ISTA para o precesso de avaliação.

\section{MATERIAL E MÉTODOS}

Para avaliar o processo de validação das duas metodologias para o teste de germinação em sementes de nabo forrageiro utilizando os substratos areia e papel com temperatura alternada $20-30{ }^{\circ} \mathrm{C}$, o trabalho foi executado em duas etapas. Na Etapa 1, denominada de procedimentos iniciais, foram coletados, selecionados e caracterizados os lotes, culminando com a distribuição dos materiais para laboratórios previamente selecionados. Esses procedimentos foram realizados no Laboratório de Análise de Sementes do Departamento de Agricultura da Universidade Federal de Lavras, MG. Na Etapa 2 foi feita uma avaliação dos resultados por meio de ferramentas estatísticas apropriadas.

\section{Etapa 1) Procedimentos iniciais}

Coleta e seleção dos lotes: Foram utilizadas sementes de sete lotes comerciais de nabo forrageiro, cultivar CATI AL 1000 de diferentes safras e procedências. As sementes de cada lote de $40 \mathrm{~kg}$ foram acondicionadas em 5 recipientes e de cada um deles foi retirada uma subamostra de $30 \mathrm{~g}$, com o auxílio de um amostrador de sementes (Brasil, 1992). Em seguida, foram realizados testes de germinação para a avaliação da homogeneidade do lote por meio do teste $\mathrm{H}$.

As subamostras foram previamente classificadas de acordo com o tamanho das sementes, utilizando-se as retidas em peneira de $8 \mathrm{~mm}$. A semeadura foi realizada em caixas em substrato papel mata-borrão umedecido com água destilada 2,5 vezes a sua massa em caixas acrílicas. Foram utilizadas 100 sementes de cada subamostra, em duas repetições de 50 em câmara de germinação tipo B.O.D. regulada à temperatura alternada $20-30{ }^{\circ} \mathrm{C}$, e procedida a contagem de plântulas normais aos três e sete dias. Com os resultados foi realizado, para cada lote, o teste H, conforme descrito na RAS (Brasil, 1992).

\section{Caracterização dos lotes - teste de germinação} inicial: Após a realização do teste $\mathrm{H}$, os cinco lotes selecionados, como os mais homogêneos e de diferentes níveis de qualidade, foram homogeneizados com o auxílio de um divisor mecânico do tipo solo com oito canaletas (Brasil, 1992). Cada lote foi dividido em oito repetições de aproximadamente $5 \mathrm{~kg}$ cada. As sementes foram classificadas de acordo com o tamanho utilizando-se uma peneira de $8 \mathrm{~mm}$. Foram testadas as duas metodologias, uma utilizando, como substrato, o papel e a outra areia nas temperaturas alternadas de $20-30^{\circ} \mathrm{C}$. A areia passou por um processo de lavagem em água corrente, esterilização em autoclave por 2 horas e umedecimento em $50 \%$ da sua capacidade de retenção, conforme Brasil (1992). O papel foi umedecido conforme já descrito no procedimento de germinação para o teste $\mathrm{H}$ e mantido em caixas acrílicas do tipo gerbox a temperatura de $20-30{ }^{\circ} \mathrm{C}$ em câmara de germinação com contagens aos três e sete dias.

As sementes dormentes, remanescentes ao final do teste, foram submetidas ao teste de tetrazólio (Brasil, 1992). Durante a contagem, as plântulas normais, plântulas anormais, sementes duras, mortas e dormentes foram descritas e fotografadas para a padronização dos resultados do teste de germinação.

O experimento, para cada metodologia, foi instalado segundo um Delineamento Inteiramente Casualizado (DIC), com 8 repetições de 50 sementes. Os dados foram submetidos à análise de variância e o efeito dos lotes, quando significativo, tiveram suas médias comparadas pelo teste de Scott-Knott, ao nível de significância de 5\%

\section{Distribuição do material para os laboratórios:}

Nessa etapa foram coletados de cada lote, amostras com cerca de $5 \mathrm{~kg}$ que foram reduzidas a $12 \mathrm{~kg}$ após classificação de acordo com o tamanho $(8 \mathrm{~mm})$. As amostras enviadas a oito laboratórios foram resultantes da subdivisão desses $1,2 \mathrm{~kg}$ ( $150 \mathrm{~g}$ por laboratório). As sementes foram acondicionadas em sacos de papel multifoliado e caixas de papelão e enviadas aos laboratórios, juntamente com as instruções para a realização e avaliação do teste de germinação, além de um questionário para avaliar a execução prática de cada uma das metodologias. Os laboratórios foram informados da data de envio das amostras, sendo solicitada a rápida execução do teste de germinação para evitar a deterioração das sementes e o envio imediato dos resultados do teste.

Deve-se ressaltar que os laboratórios selecionados ou eram de prestação de serviço ou de pesquisa, credenciados ou não pelo Ministério de Agricultura, 
Pecuária e Abastecimento (MAPA) e com diferentes níveis de experiência com o teste de germinação para nabo forrageiro.

\section{Etapa 2) Avaliação dos resultados}

Esta etapa foi subdivida em 6 subetapas, sendo que as técnicas estatísticas foram utilizadas para: identificar a presença de valores discrepantes e outliers nas variâncias; avaliar os efeitos de laboratórios e lotes; verificar a precisão (repetibilidade e reprodutibilidade), exatidão e robustez; comparar as diferentes metodologias de germinação e reavaliar o nível de qualidade dos lotes. Cada etapa foi realizada separadamente para os substratos papel e areia, com exceção dos testes $\mathrm{F}$ que foram utilizados justamente para comparar os resultados das duas metodologias.

Identificação de valores discrepantes (outlier): Para a detecção de valores discrepantes em todos os laboratórios foi utilizado o método de Hampel (Hampel, 1985). A orientação do programa de validação nos casos em que for detectado algum outlier é promover a sua retirada do banco de dados, antes de proceder às próximas análises (ISTA, 2007).

Identificação de outlier nas variâncias: Em cada lote foi realizado o teste de Levene utilizando a média (Levene, 1960). No caso da detecção a presença de outlier, o laboratório com maior variância foi retirado e o processo repetido, até que ficassem apenas os laboratórios com variâncias homogêneas de acordo com o teste Levene.

Análise de Variância: Para avaliar os efeitos dos laboratórios e lotes, foi aplicada a teoria da análise de variância num delineamento inteiramente casualizado em esquema fatorial com 8 repetições de 50 sementes cada. Ressalta-se que no caso deste estudo, não havia razões práticas para avaliar o efeito da interação entre laboratórios e lotes, assim este fator não foi explicitado diretamente no modelo.

Avaliação da precisão (Repetibilidade e Reprodutibilidade) e das estatísticas h e k de Mandel: A variância de repetibilidade $\left(S_{r}^{2}\right)$ representa uma medida da variabilidade dentro dos laboratórios (ISO 5725-2, 1994). Com seu valor determinado, foi calculado o limite crítico $(r)$ de repetibilidade, estimado por $r=S_{r}$ $D$, em que $D$ foi obtido da tabela de Tukey, com graus de liberdade tendendo para o infinito e grau de confiança de $99 \%$ (Banzatto e Konkra, 2006). Esse valor de $r$ foi comparado com a amplitude entre as repetições de cada laboratório, em cada lote, avaliando-se, então, quais laboratórios tiveram repetibilidade aceitável.

A variância de reprodutibilidade $\left(S_{R}^{2}\right)$ representa uma medida da variabilidade entre e dentro dos laboratórios (ISO 5725-2, 1994), e com seu valor determinado foi calculado o limite crítico $(R)$ de reprodutibilidade para cada lote, estimado por $R=S_{R} D$. Esse valor de $R$ foi comparado com a amplitude entre os laboratórios para cada repetição, determinando, assim, quais lotes tiveram reprodutibilidade aceitável, com grau de confiança de $99 \%$.

Outra medida utilizada para auxiliar na avaliação da precisão dos resultados foi a estatística $\mathrm{k}$ de Mandel, já a estatística $h$ foi a medida utilizada para avaliar graficamente a estimativa do viés, por conseguinte a exatidão, dos resultados (ISO 5725-2, 1994). Após os cálculos, foram esboçados os gráficos dos valores de $h$ e k para cada lote e laboratório, e comparados com os valores críticos para os níveis de significância de $1 \%$ e $5 \%$.

Comparação das metodologias: Para a comparação da precisão das metodologias dentro dos laboratórios foi calculada uma estatística $\mathrm{F}_{r j}$ para cada lote $(j=1, \ldots, 5)$ e feita a comparação com dois limites tabelados $F_{r j}$ inf $\mathrm{e}_{r j \text { sup }}$ utilizando-se as estimativas das variâncias da repetibilidade considerando os dois substratos, areia e papel (Lupin; Schouenborg, 2000). As linhas de decisão foram assim definidas: se $F_{r j}>F_{r j \text { inf }} \geq F_{r j} \geq F_{r j \text { sup }}$ não há evidências de que as metodologias tenham precisões diferentes dentro de cada laboratório; se $\mathrm{F}_{r j}>\mathrm{F}_{r j \text { sup }}$ há evidências de que a metodologia 1 tenha precisão superior a metodologia 2 dentro de cada laboratório; e se $F_{r j}>F_{r j \text { sup }}$ há evidências de que a metodologia 1 tenha precisão inferior a metodologia 2 dentro de cada laboratório.

$\mathrm{Na}$ comparação da precisão geral das metodologias foi calculada uma estatística $F_{R j}$ para cada lote $(j=1, \ldots, 5)$ e feita a comparação com os valores críticos, similares aos apresentados para a comparação da precisão das metodologias dentro dos laboratórios, bem como as linhas de decisão. A única diferença é que a interpretação foi feita para a precisão geral, sendo então utilizadas as estimativas das variâncias de reprodutibilidade e repetibilidade dos substratos areia e papel (Lupin; Schouenborg, 2000):

Classificação dos lotes: Como foram selecionados lotes com níveis de qualidade diferentes utilizou-se a técnica de Análise Discriminante para averiguar, baseados nos resultados obtidos pelos laboratórios, a adequação da classificação feita a priori. Assim, foram formados três grupos, correspondentes aos lotes de qualidade inferior, 
intermediária e superior.

$\mathrm{O}$ uso desta técnica consistiu em determinar uma combinaçãolinearentreduasoumais variáveisindependentes que discriminaram melhor os grupos definidos a priori. A discriminação foi conseguida estabelecendo-se os pesos da combinação linear que maximizaram a variância entre grupos relativos à variância dentro dos grupos. A combinação linear para a Análise Discriminante é também conhecida como função discriminante (Hair et al., 2006). Uma vez que a função discriminante linear foi calculada, uma observação pôde ser alocada para uma das $g$ populações com base em seu "score discriminante", e como na amostra, a população de origem de cada observação era conhecida, foi possível identificar quais deles foram classificados corretamente ou não. Assim, pôde ser calculada a Taxa Estimada de Erro (TEE), que corresponde a fração das observações incorretamente classificadas, sendo uma estimativa da Taxa de Erro verdadeira.

Ressalta-se que o procedimento padrão da ISTA para o processo de validação de metodologia indica o uso dos métodos de Hampel, a Análise de Variância, os valores de repetibilidade e reprodutibilidade, e as estatísticas h e $\mathrm{k}$ de Mandel. Já as técnicas estatísticas complementares propostas neste trabalho foram: o teste de Levene para média (em substituição ao teste de Cochran), os limites críticos de repetibilidade, os testes $\mathrm{F}$ para comparação das metodologias e a Análise Discriminante.

Para todas as análises estatísticas foram desenvolvidas funções específicas no software $\mathrm{R}$ ( $\mathrm{R}$ Development Core Team, 2008).

\section{RESULTADOS E DISCUSSÃO}

De acordo com os resultados do teste $\mathrm{H}$, cinco dos sete lotes avaliados, não apresentaram evidências estatísticas para rejeitar a hipótese de homogeneidade do lote, ao nível de significância de 1\% de acordo com Brasil (1992) e Copeland e Liu (2005).

\section{Caracterização dos lotes - Teste de germinação inicial}

Pelos resultados obtidos no teste de germinação foram observadas diferenças significativas para o efeito de lotes tanto na utilização do substrato areia como papel, pelo teste de Scott-Knott, no nível de significância de 5\% (Tabela 1). De acordo com as diferenças apresentadas nesse teste, os lotes foram classificados, quanto a porcentagem de germinação, em diferentes níveis de qualidade fisiológica: inferior, intermediária e superior. A exceção foi o lote 5, para a metodologia papel, que apesar da porcentagem de germinação ter sido considerada estatisticamente igual a dos lotes 1 e 3, o mesmo foi classificado como nível intermediário, em razão do resultado obtido na areia e por apresentar valor médio de germinação entre 60 a $70 \%$, próximo ao valor mínimo para comercialização de sementes da espécie Raphanus sativus que era de $60 \%$ (Brasil, 2009).

TABELA 1. Resultados médios (\%) obtidos no teste de germinação inicial com sementes de 5 lotes de nabo forrageiro utilizando os substratos areia e papel com temperatura alternada de 20-30 ${ }^{\circ} \mathrm{C}$.

\begin{tabular}{ccccc}
\hline \multirow{2}{*}{ Lote } & \multicolumn{2}{c}{ Areia } & Papel \\
\cline { 2 - 5 } & ${ }^{1}$ Médias & Classificação & 'Médias & Classificação \\
\hline 1 & $76 \mathrm{a}$ & Superior & $75 \mathrm{a}$ & Superior \\
2 & $55 \mathrm{c}$ & Inferior & $44 \mathrm{c}$ & Inferior \\
3 & $76 \mathrm{a}$ & Superior & $70 \mathrm{a}$ & Superior \\
4 & $66 \mathrm{~b}$ & Intermediária & $57 \mathrm{~b}$ & Intermediária \\
5 & $67 \mathrm{~b}$ & Intermediária & $67 \mathrm{a}$ & Intermediária \\
\hline
\end{tabular}

${ }^{1}$ Médias seguidas de mesma letra minúscula na coluna não diferem entre si pelo teste de Scott-Knott, no nível de significância de 5\%.

Para Waeny (1980), a utilização de lotes com níveis de qualidades diferentes no processo de validação de metodologias é necessária, porque a metodologia ideal deve ser robusta para suportar condições adversas de aplicação e, também, ser precisa e exata para detecção de diferenças sutis de qualidade.

\section{Teste de germinação nos laboratórios selecionados}

Observa-se pelo resultado geral dos oito laboratórios, que para todos os lotes no substrato areia, a média de 
germinação foi maior e o coeficiente de variação foi menor em relação ao papel (Tabela 2). Resultados similares, sob as mesmas condições de teste, foram encontrados por Nery et al. (2007) e Nery (2008).

TABELA 2. Resultados médios percentuais $(\bar{y})$ e coeficientes de variação (CV\%) obtidos em 8 laboratórios para o teste de germinação com sementes de 5 lotes de nabo forrageiro em substratos areia e papel, com temperatura alternada de $20-30{ }^{\circ} \mathrm{C}$.

\begin{tabular}{|c|c|c|c|c|c|c|c|c|c|c|c|}
\hline \multirow{2}{*}{ Substrato } & \multirow{2}{*}{ Lab. } & \multicolumn{2}{|c|}{ Lote1 } & \multicolumn{2}{|c|}{ Lote 2} & \multicolumn{2}{|c|}{ Lote 3} & \multicolumn{2}{|c|}{ Lote 4} & \multicolumn{2}{|c|}{ Lote 5} \\
\hline & & $\bar{y}$ & $\mathrm{CV}$ & $\bar{y}$ & $\mathrm{CV}$ & $\bar{y}$ & $\mathrm{CV}$ & $\bar{y}$ & $\mathrm{CV}$ & $\bar{y}$ & $\mathrm{CV}$ \\
\hline \multirow{9}{*}{ Areia } & 1 & 89 & 4,90 & 55 & 8,33 & 87 & 6,54 & 64 & 0,91 & 80 & 3,31 \\
\hline & 2 & 94 & 4,87 & 62 & 6,52 & 95 & 1,61 & 78 & 5,18 & 87 & 2,90 \\
\hline & 3 & 98 & 0,59 & 64 & 6,31 & 96 & 0,00 & 85 & 1,18 & 92 & 0,00 \\
\hline & 4 & 94 & 2,81 & 62 & 7,95 & 94 & 3,07 & 71 & 16,32 & 85 & 4,25 \\
\hline & 5 & 92 & 2,51 & 68 & 5,94 & 95 & 1,05 & 72 & 9,11 & 86 & 4,08 \\
\hline & 6 & 92 & 4,52 & 63 & 11,14 & 91 & 6,25 & 66 & 18,08 & 79 & 5,80 \\
\hline & 7 & 91 & 6,34 & 61 & 8,67 & 93 & 3,11 & 69 & 8,25 & 85 & 0,68 \\
\hline & 8 & 93 & 5,69 & 63 & 16,06 & 91 & 12,38 & 79 & 14,38 & 85 & 13,94 \\
\hline & Geral & 93 & 4,51 & 62 & 9,60 & 93 & 5,45 & 73 & 12,90 & 86 & 6,31 \\
\hline \multirow{9}{*}{ Papel } & 1 & 88 & 4,55 & 53 & 17,02 & 89 & 2,83 & 59 & 11,29 & 83 & 5,52 \\
\hline & 2 & 91 & 4,96 & 56 & 8,80 & 94 & 1,06 & 61 & 14,13 & 85 & 3,78 \\
\hline & 3 & 98 & 0,59 & 68 & 3,06 & 93 & 0,62 & 83 & 3,69 & 94 & 2,13 \\
\hline & 4 & 94 & 4,87 & 61 & 11,16 & 94 & 1,06 & 65 & 19,66 & 85 & 9,51 \\
\hline & 5 & 95 & 2,19 & 66 & 10,61 & 96 & 1,59 & 81 & 7,44 & 93 & 4,06 \\
\hline & 6 & 92 & 3,49 & 61 & 4,34 & 84 & 9,55 & 68 & 13,26 & 77 & 8,45 \\
\hline & 7 & 76 & 8,36 & 40 & 29,63 & 74 & 14,04 & 45 & 37,47 & 60 & 20,88 \\
\hline & 8 & 95 & 1,05 & 64 & 8,27 & 95 & 1,61 & 77 & 8,52 & 88 & 0,66 \\
\hline & Geral & 91 & 7,78 & 59 & 17,54 & 90 & 9,19 & 68 & 21,49 & 83 & 13,95 \\
\hline
\end{tabular}

Nos casos de espécies com nível de domesticação inferior, ou com altos índices de infestação com microrganismos, segundo Ashton (2005), recomenda-se a utilização do substrato areia, uma vez que este material possibilita uma maior taxa e velocidade de germinação. A maior velocidade de germinação pode ser explicada por autores tais como, Lopes et al., (2005); Pacheco et al., 2006); Nery, (2008), como sendo consequência da maior área de contato da semente com a areia, o que propicia maior velocidade na absorção de água. Da mesma forma, as menores médias de germinação obtidas no substrato papel parecem estar associadas, segundo Andrade et al. (2006), a uma menor capacidade de retenção de água de alguns materiais ou a proximidade entre plântulas no substrato o que pode facilitar a contaminação por microrganismos. No entanto os resultados podem variar conforme o lote e a espécie analisada (Kopper et al, 2010).

Para as duas metodologias avaliadas, o lote 2 no geral apresentou menores médias de germinação em relação aos demais lotes, o que corrobora com a classificação estabelecida no teste de germinação inicial, como sendo este de qualidade inferior. Em relação ao coeficiente de variação, no geral, observa-se maiores valores para os lotes 2, 4 e 5 que foram considerados em estados mais avançados de deterioração. Resultados semelhantes foram encontrados em Illipront (1997) trabalhando com sementes de soja, que concluiu existir maior variação individual de sementes em lotes com estados progressivos de deterioração em relação aos deteriorados ou aqueles de qualidade superior.

Com a utilização do substrato areia foi identificado pelo método de Hampel uma quantidade menor de outliers num total de três, em relação ao papel, onde foram observados 
sete outliers. De acordo com Williams et al. (2002) e Liu et al. (2004) é importante a identificação dos valores discrepantes antes da modelagem e análise, pois a presença destes valores podem levar a um modelo mal especificado, a estimação viesada de parâmetros, e, por conseguinte, a resultados incorretos.

Foram identificados pelo teste de Levene, no nível de significância de $1 \%$, outliers nos lotes 3 (valor $p=0,0099$ ), 4 (valor $\mathrm{p}=0,0067$ ) e 5 (valor $\mathrm{p} 0,0009$ ) para o substrato areia e o lote 3 (valor $p=0,0073$ ) para o papel. Para cada lote em que foram identificados outliers, retirou-se o laboratório com maior variância, que foram: laboratórios 4 e 7 do lote 3; laboratório 6 do lote 4 ; laboratório 8 do lote 5 para o substrato areia e o laboratório 6 do lote 3 para o papel.

Após a retirada dos laboratórios repetiu-se o teste de
Levene e, com exceção do lote 4 (valor $p=0,0080$ ), para os demais lotes não foi rejeitada a hipótese nula de variâncias homogêneas nos laboratórios restantes. Assim, para o lote 4 foi necessário reaplicar o teste de Levene pela segunda vez, sendo excluído também o laboratório 4. E, finalmente, as variâncias dos laboratórios restantes para este lote foram consideradas homogêneas (valor $\mathrm{p}=0,0145$ ).

Com as retiradas de outliers, o delineamento experimental passou a ser não balanceado, logo, para a obtenção dos quadrados médios foram utilizadas as somas de quadrados tipo III. Pelos resultados da análise de variância, no nível de significância de $1 \%$, foram identificadas, para as duas metodologias, diferenças estatisticamente significativas entre os resultados de diferentes laboratórios (Tabela 3).

TABELA 3. Resumo da análise de variância para a variável porcentagem de germinação em substrato areia e papel, com temperatura alternada de $20-30{ }^{\circ} \mathrm{C}$, de acordo com oito laboratórios, em ensaios com sementes de cinco lotes de nabo forrageiro.

\begin{tabular}{|c|c|c|c|c|c|}
\hline Sub. & Fonte de variação & G.L. & Quadrado médio & $\mathrm{F}$ & Valor $\mathrm{p}$ \\
\hline \multirow{4}{*}{ Areia } & Laboratório & 7 & 122,8865 & 5,27 & $<0,0001$ \\
\hline & Lote & 4 & $3.912,8757$ & 167,94 & $<0,0001$ \\
\hline & Resíduo & 90 & 23,2987 & & \\
\hline & C.V $(\%)$ & & 5,94 & & \\
\hline \multirow{4}{*}{ Papel } & Laboratório & 7 & 717,1304 & 19,05 & $<0,0001$ \\
\hline & Lote & 4 & $4.588,0339$ & 121,87 & $<0,0001$ \\
\hline & Resíduo & 99 & & & \\
\hline & C.V $(\%)$ & & 7,78 & & \\
\hline
\end{tabular}

Algumas hipóteses podem ser levantadas para explicar tais diferenças nos resultados do teste de germinação, como, por exemplo, a alta variação no estádio de maturação comum em sementes de nabo forrageiro (Bittencourt et al., 1991). Uma possível dificuldade na análise e interpretação do teste pelos laboratórios por ser uma planta pouco avaliada e conhecida pelos mesmos, ou ainda, o excesso de microrganismos associados a essa espécie, (Nery, 2008).

\section{Avaliação da repetibilidade, reprodutibilidade e dos valores de $\mathbf{h}$ e $\mathbf{k}$}

Comparando a amplitude de cada laboratório com os limites críticos de repetibilidade por lote, grau de confiança de $99 \%$, pôde-se verificar que, no caso da areia com exceção dos laboratórios 4 e 7 em relação ao lote 3 e laboratório 8 para o lote 5 , todos os demais apresentaram repetibilidade aceitável. De fato, esses laboratórios que não apresentaram repetibilidade não tinham sido incluídos no cálculo do limite crítico, já que foram retirados da análise em etapas anteriores, sendo reapresentado apenas para confirmar a situação de não adequabilidade dos seus resultados, para os lotes mencionados, no processo de validação. Já para o substrato papel, as exceções foram os laboratórios 6 e 7 para o lote 3 , que também tinham sido retirados da análise nas outras etapas.

Quanto às condições de reprodutibilidade no caso da areia, comparando a amplitude de cada repetição com os limites críticos por lote, grau de confiança de $99 \%$, pôde-se verificar que todos os lotes apresentaram reprodutibilidade aceitável. No caso do substrato papel, a exceção foi novamente o lote 4. Pela observação dos gráficos dos valores de h e k (Figura 1) para o substrato areia, verifica-se que os h e $\mathrm{k}$ críticos a $1 \%$ 
não foram ultrapassados, evidenciando a consistência dos resultados de cada lote por laboratório, ocorrendo apenas uma tendência a subestimação pelo laboratório 1 e superestimação pelo laboratório 3 comparados com a média geral, de acordo com os valores de h; e uma maior variabilidade entre os lotes para o laboratório 8 de acordo com os valores de $\mathrm{k}$.
Para o substrato papel verifica-se também que os $\mathrm{h}$ e $\mathrm{k}$ críticos a $1 \%$ não foram ultrapassados (Figura 2), com uma tendência a subestimação dos resultados pelos laboratórios 1 e 7, superestimação pelos laboratórios 3 e 5 comparados com a média geral, baseado nos valores de h; e maior variabilidade entre os lotes dos laboratórios 1 e 7 de acordo com os valores de $\mathrm{k}$.
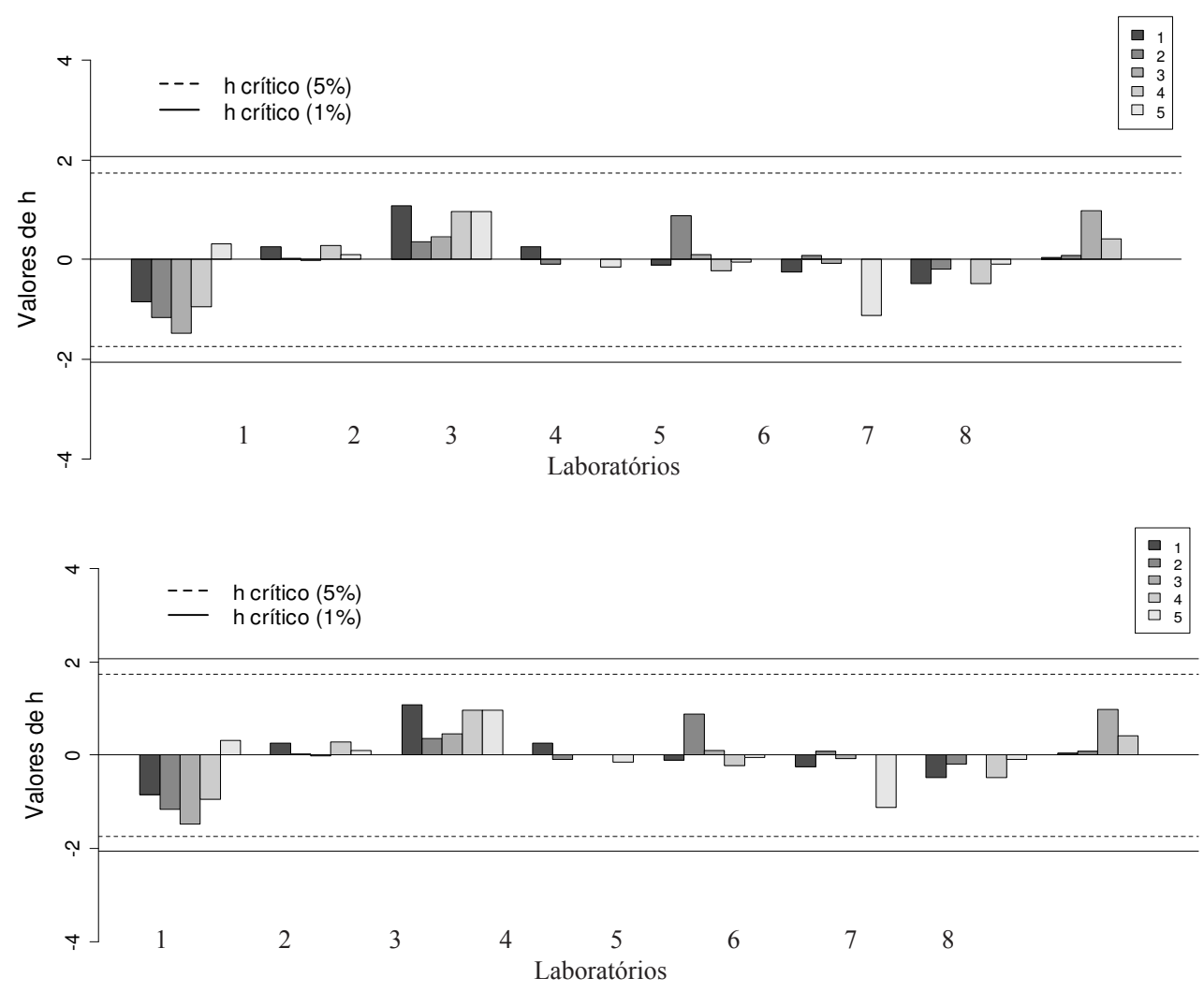

FIGURA 1. Gráficos dos valores de h e k para o teste de germinação em sementes de nabo forrageiro em substrato areia e temperatura alternada de $20-30{ }^{\circ} \mathrm{C}$.

\section{Comparação das metodologias}

De acordo com o teste $\mathrm{F}$ não existiram evidências estatísticas para considerar diferenças na precisão nem dentro dos laboratórios e nem no geral para as duas metodologias em cada lote. Além dos resultados estatísticos, cada laboratório preencheu uma ficha de avaliação (questionário) comparando as duas metodologias, sempre indicando qual das duas seria mais fácil na execução de alguns procedimentos, como: pré-montagem, montagem, manipulação e avaliação. Para todas as respostas a metodologia papel foi eleita pela maioria dos laboratórios como a mais fácil.

\section{Análise Discriminante}

Para avaliar a função de classificação foi utilizado o processo de validação cruzada, obtendo-se as matrizes de confusão para as duas metodologias (Tabelas 4 e 5). Com os resultados das matrizes de confusão obtiveramse, para as duas metodologias, as três taxas de erro de se classificar: no geral uma observação incorretamente - TEE; uma observação do grupo $t_{i}$ no grupo $t_{j}-$ TEE $(j \mid i)$, e uma observação do grupo $t_{i}$ incorretamente - TEE ( $i$ ) (Tabela 6). Para a metodologia areia, somente o grupo 2 apresentou uma taxa de erro de classificação, o que era em parte esperado por serem lotes de qualidade intermediária. 

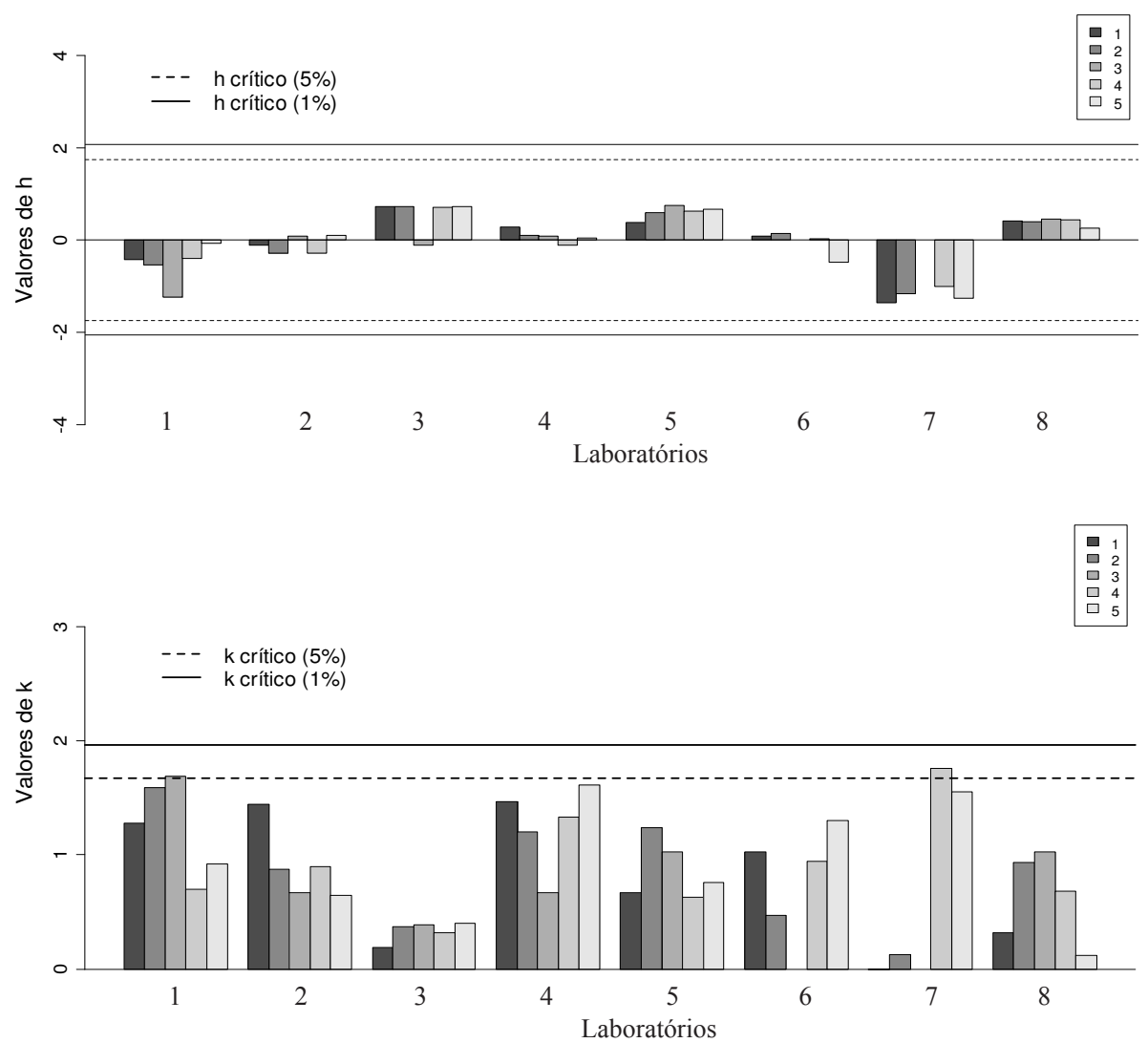

FIGURA 2. Gráficos dos valores de h e k para o teste de germinação em sementes de nabo forrageiro em substrato papel, na temperatura alternada de $20-30{ }^{\circ} \mathrm{C}$.

TABELA4. Matriz de confusão com validação cruzada - regra linear de Fisher, para os resultados do teste de germinação em sementes de nabo forrageiro utilizando substrato areia e temperatura alternada de $20-30{ }^{\circ} \mathrm{C}$.

\begin{tabular}{ccccc}
\hline Grupo & \multicolumn{3}{c}{ Grupo classificado } & total \\
\cline { 2 - 4 } verdadeiro & $\tau_{1}$ & $\tau_{2}$ & $\tau_{3}$ & 8 \\
$\tau_{1}$ & 8 & 0 & 0 & 13 \\
$\tau_{2}$ & 2 & 8 & 3 & 14 \\
$\tau_{3}$ & 0 & 0 & 14 & 35 \\
Total & 10 & 8 & 17 & \\
\hline
\end{tabular}

TABELA 5. Matriz de confusão com validação cruzada - regra linear de Fisher, para os resultados do teste de germinação em sementes de nabo forrageiro em substrato papel e temperatura alternada de $20-30{ }^{\circ} \mathrm{C}$.

\begin{tabular}{ccccc}
\hline Grupo & \multicolumn{3}{c}{ Grupo classificado } & Total \\
\cline { 2 - 5 } verdadeiro & $\tau_{1}$ & $\tau_{2}$ & $\tau_{3}$ & 8 \\
$\tau_{11}$ & 6 & 2 & 0 & 16 \\
$\tau_{2}$ & 3 & 6 & 7 & 15 \\
$\tau_{3}$ & 0 & 2 & 13 & 39 \\
Total & 9 & 10 & 20 & \\
\hline
\end{tabular}


Pelos resultados apresentados na matriz de confusão para o substrato areia (Tabela 4), verifica-se uma tendência do grupo 2 tanto a sub como super estimação, uma vez que foram duas observações classificadas no grupo 1 , e três no grupo 3 . E desses cinco valores confundidos, quatro deles são observações do lote 4 , reforçando também o que já foi dito sobre este lote, no que se refere a sua alta variabilidade em relação aos demais lotes. Comparando as duas metodologias, verifica-se que os três tipos de TEE para o substrato papel foram bem superiores ao da areia, evidenciando um fato já constatado de uma variação maior dos resultados obtidos no papel. Segundo Ferreira (2008), o desempenho de uma regra de classificação depende das distâncias dos grupos, e essa distância depende da variabilidade, quanto menor for a dispersão do grupo, menores serão os erros de classificação incorreta.

TABELA 6. Taxas de erro (\%) para classificações pelo processo de validação cruzada - regra linear para o teste de germinação em sementes de nabo forrageiro em substrato areia e temperatura alternada de $20-30{ }^{\circ} \mathrm{C}$.

\begin{tabular}{ccc}
\hline $\begin{array}{c}\text { Taxas de } \\
\text { erro }\end{array}$ & Areia & Papel \\
\hline TEE & 14,29 & 35,90 \\
\hline TEE $(1 \mid 2)$ & 15,38 & 18,75 \\
TEE $(1 \mid 3)$ & 0,00 & 0,00 \\
TEE $(2 \mid 1)$ & 0,00 & 25,00 \\
TEE $(2 \mid 3)$ & 0,00 & 13,33 \\
TEE $(3 \mid 1)$ & 0,00 & 0,00 \\
TEE $(3 \mid 2)$ & 23,08 & 43,75 \\
\hline TEE $(1)$ & 0,00 & 18,75 \\
TEE $(2)$ & 38,46 & 62,50 \\
TEE (3) & 0,00 & 15,38 \\
\hline
\end{tabular}

Pode se observar que, independente da metodologia, o grupo 2 apresentou as maiores taxas de erro de classificação, o que parece estar associado a uma maior dispersão nos resultados obtidos num lote de qualidade intermediária. Como afirma Ferreira (2008), "por melhores que sejam os métodos matemáticos uma boa regra de decisão dependerá de outros fatores exógenos ao controle."

\section{CONCLUSÕES}

As técnicas estatísticas associadas à validação propostas como complementares ao padrão da ISTA, contribuíram para a melhoria e aperfeiçoamento das análises no processo de validação e permitem afirmar que:

$\mathrm{O}$ uso do teste de Levene para média possibilita identificar laboratórios discrepantes que não seriam considerados com o teste de Cochran.

As condições dos limites críticos de repetibilidade e reprodutibilidade e robustez pode ser observadas em dados obtidos na avaliação da germinação de sementes de lotes de nabo forrageiro de diferentes níveis de qualidade.

A técnica da Análise Discriminante auxilia o processo de validação de metodologia, confirmando, com os resultados obtidos nos laboratórios, a classificação de um lote quanto ao seu potencial de germinação.

As metodologias para o teste de germinação em sementes de nabo forrageiro com a utilização dos substratos areia e papel com temperatura alternada $20-30^{\circ} \mathrm{C}$, puderam ser consideradas validadas, tanto no aspecto fitotécnico, como estatístico.

\section{AGRADECIMENTOS}

À FAPEMIG (Fundação de Amparo à Pesquisa do Estado de Minas Gerais) e ao CNPq (Conselho Nacional de Desenvolvimento Científico e Tecnológico), pelo suporte financeiro e ao MAPA ( Ministério da Agricultura, Pecuária e Abastecimento), pela distribuição das sementes aos laboratórios credenciados.

\section{REFERÊNCIAS}

ANDRADE, A.C.S.; PEREIRA,T.S.; FERNANDES.; M.J.; CRUZ,A.P.M.; CARVALHO, A.S.R. Substrato, temperatura de germinação e desenvolvimento pós-seminal de sementes de Dalbergia nigra. Pesquisa Agropecuária Brasileira, v.41, n.3, p.517-523, 2006.

ASHTON, P.M. Seed Germinatio Testing. In: Seed Technologists Training Manual. The Society of Commercial Seed Technologists, 2005. 450p.

ASSOCIATION OF OFFICILASEED ANALISTS. Seed vigour testing handbook. East Lansing: AOSA, 1983. 88p. (Contribution, 32).

BANZATTO, D.A.; KONKRA, S.N. Experimentação Agrícola. 4.ed. Jaboticabal: FUNEP. 2006. 237p. 
BITTENCOURT, J.F.N.; SADER, R.; UNAGRO, M.R.G.; TOLEDO, N.M. P. Maturação fisiológica de sementes de girasol cv. Contiso. Revista Brasileira de Sementes, v.13, n.2, p.81-85, 1991.

BRASIL. Ministério da Agricultura e Reforma Agrária. Secretaria Nacional de Defesa Agropecuária. Departamento Nacional de Produção Vegetal. Coordenação de Laboratório Vegetal. Regras para Análise de Sementes. Brasília, DF, 1992. 365p.

BRASIL, Minestério da Agricultura, Pecuária e Abastecimento. Instrução Normativa $\mathbf{N}^{\mathbf{3}}$ 30. 2008. Disponível em: $<$ http://www.puntofocal.gov.ar/notific otros_miembros/bra288_t.pdf $>$. Acesso em: 2 jan. 2009.

COPELAND, L.; LIU, H. Seed Sampling and Sub sampling. In: Seed Technologists Training Manual. The Society of Commercial Seed Technologists, 2005. 450p.

EURACHEM, G. The fitness for purpose of analytical methods. a laboratory guide to method validation and related topics, UK, 1998.

FERREIRA, D.F. Estatística Multivariada. Lavras: UFLA, 2008. 661p.

HAIR, J.F.Jr.; ANDERSON, R.E.; TATHAN, R.L.; BLACK, W.C. Multivariate Data Analysis. 5.ed. Upper Saddle River: Prentice Hall. Tradução: Pearson Education. 2006. 593p.

HAMPEL, F.R. The breakdown points of the mean combined with som rejection rules. Technometrics, n.27, p.95-107,1985.

ILLIPRONTI, JUNIOR, R.A. Variation in quality of individual seeds within a seed loto f soyeban (Glycine Max (L.) Merrill). 1997. 157f. Thesis (Doctoral) Wageningen Agricultural University, Wageningen, 1997.

ISTA. Method Validation for Seed Testing. International Seed Testing Association, Switzerland, 2007.

ISO 5725/2. Accuracy (trueness and precision) of measurement methods and results - Part 2: Basic method for the determination of repeatability and reproducibility of a standard measurement method. International Organization for Standardization, Genève, 1994.

KOPPER, A.C; MALAVASI, M. M; MALAVASI, U.C. Influência da temperatura e do substrato na germinação de sementes de Cariniana strellensis (Raddi) Kuntze. Revista Brasileira de Sementes, vol32, n.2, p160-165, 2010.

LEVENE, $\mathrm{H}$. Robust tests for equality of variances. In: OLKIN, I.; GHURYE, S.G.; HOEFFDING, W.; MADOW,
W.G.; MANN, H.B. (Ed.).Contributions to Probability and Statistics: essays in honor of harold hotelling. Stanford University Press, p.278-292, 1960.

LIU, H.; SHAH, S.; JIANG, W. On-line outlier detection and data cleaning. Computers and Chemical Engineering, United Kingdom, n. 28, p. 1635-1647, 2004.

LOPES, J.C.; CAPUCHO, M.T.; MARTINS FILHO, S.; REPOSSI, P.A. Influência da temperatura, substrato e luz na germinação de sementes de bertalha. Revista Brasileira de Sementes. v.27, n.2, p.18-24, dez. 2005.

LUPING, T.; SCHOUENBORG, B. Methodology of Inter-comparison Tests and Statistical Analysis of the results. SP Swedish National Testing and Research Institute. SP REPORT. 2000. p.35. Disponível em: <http:// www.nordicinnovation.net/nordtestfiler/tec482.pdf $>$. Acesso em: jan. 2008.

NERY, M.C.; CARVALHO, M.L.M. de.; GRIS, C.F.; NERY, F.C.; LEAL FILHO, W.R.; FRAGA, A.C. Germinação de sementes de nabo forrageiro cultivar IPR 116. In: Congresso Brasileiro de Plantas Oleaginosas, Óleos, Gorduras e Biodiesel, 4., Resumos... Varginha, 2007. 1CD-ROM.

NERY, M.C. Colheita, beneficiamento e controle de qualidade de sementes de nabo forrageiro 2008. $180 \mathrm{f}$. Tese (Doutorado em Fitotecnia) - Universidade Federal de Lavras, Lavras 2008.

PACHECO, M.V.; MATOS,V.P.; FERREIRA, R.L.; FELICIANO, A.L.P.; PINTO, K.M.S. Efeito de temperatura e sibstratos na germinação de sementes de Myracrodruon urundeuva Fr. All, (Anarcadiace) Revista Árvore, v.30, n.3, p.359-367, maio/jun. 2006.

$\mathrm{R}$ Development Core Team. R: a language and environment for statistical computing. $R$ Foundation for Statistical Computing, Vienna, Áustria 2008. Disponível em: $<$ http://www.R-project.org $>$. Acesso em: 2 set. 2010.

SHEPPARD, J.W.; COCKERELL, V. International Seed Testing Association Handbook of Method Validation for the Detection of Seedborne Pathogens. 2000.

WAENY, J.C.C. Repetitividade e reprodutividade II. São Paulo: IPT. 1980. 14p.

WILLIAMS, G.J.; BAXTER, R.A.; He H.X.; HAWKINS S.; Gu L. A Comparative Study of RNN for Outlier Detection in Data Mining IEEE International Conference on Data-mining (ICDM'02), Maebashi City, Japan, CSIRO Technical Report CMIS-02/102. 2002. 